\title{
The Influence of Drinking Pattern, at Individual and Aggregate Levels, on Alcohol-Related Negative Consequences
}

\author{
M. Astudillo ${ }^{a}$ S. Kuntsche ${ }^{a} \quad$ K. Graham ${ }^{c} \quad$ G. Gmel ${ }^{a, b}$ \\ a Swiss Institute for the Prevention of Alcohol and Drug Problems, and ${ }^{b}$ Alcohol Treatment Center, Lausanne \\ University Hospital, Lausanne, Switzerland; ' ${ }^{C}$ Center for Addiction and Mental Health, London Ont., Canada
}

\section{Key Words}

Alcohol consumption $\cdot$ Cultural aspects $\cdot$ Drinking patterns

\begin{abstract}
Aim: To determine the extent drinking patterns (at the individual and country level) are associated with alcohol-related consequences over and above the total alcohol the person consumes. Methods: Hierarchical linear models were estimated based on general population surveys conducted in 18 countries participating in the GENACIS project. Results: In general, the positive association between drinking pattern scores and alcohol-related consequences was found at both the individual and country levels, independent of volume of drinking. In addition, a significant interaction effect indicated that the more detrimental the country's drinking pattern, the less steep the association between the volume of drinking and its consequences. Conclusion: Drinking patterns have an independent impact on consequences over and above the relationship between volume and consequences.
\end{abstract}

Copyright $\odot 2010$ S. Karger AG, Basel

\section{Introduction}

There is no doubt that the consumption of alcoholic beverages increases the risk of experiencing negative consequences [1]. Overall alcohol use has been linked to more than 200 disease conditions [2]. To explore this link, the long-standing tradition in alcohol epidemiology has been to focus on total overall alcohol consumption (sometimes referred to as volume of drinking). Over the last two decades, however, greater attention has been given to the effects of drinking pattern on negative consequences over and above the effects from total alcohol consumption [1, $3,4]$.

Drinking pattern is important because the way alcohol is consumed has differential effects given the same volume (e.g. the differential impact of drinking 1 drink every day for 7 days vs. 7 drinks in 1 day). The most studied aspect of drinking pattern has been risky single-occasion drinking (RSOD, also called 'binge' drinking or heavy episodic drinking). Definitions of RSOD vary among studies [e.g. 5 and 6], but RSOD tends to be defined as 60 to $70 \mathrm{~g}$ of pure ethanol consumed on a single occasion (sometimes less for women). RSOD has been linked with the occurrence of a number of different types of harm to self and others, including increased risk of negative social consequences, injuries, driving under the influence of alcohol, sexually transmitted diseases and coronary heart disease [see 7, 8 for overviews]. Links between RSOD and acute consequences from drinking have been especially evident. For instance, one study [9] of alcohol-related injury found not only that injury increased at higher levels of volume of drinking, but also at a given volume of drinking, those who engaged in RSOD were at

\section{KARGER \\ Fax +4161306 1234 \\ E-Mail karger@karger.ch}

(C) 2010 S. Karger AG, Basel

www.karger.com
Accessible online at:

www.karger.com/ear
Mariana Astudillo

Swiss Institute for the Prevention of Alcohol and Other Drug Problems

PO Box 870

CH-1001 Lausanne (Switzerland)

E-Mail mastudillo@sfa-ispa.ch 
Table 1. Criteria for the drinking pattern scale used for CRA and in analyses by Gmel et al. [4]

\section{Link to disease burden}

$\begin{array}{ll}\begin{array}{l}\text { Usual quantity } \\ \text { per occasion }\end{array} & \begin{array}{l}\text { Heavy drinking occasions (RSOD) lead to in- } \\ \text { creases in injuries [34] and have been shown to }\end{array} \\ \begin{array}{l}\text { Festive drinking* } \\ \text { Frequency of }\end{array} & \begin{array}{l}\text { lead to detrimental cardiovascular outcomes } \\ \text { getting drunk }\end{array} \\ \begin{array}{l}\text { Ceteris paribus, the higher the frequency of } \\ \text { (RSOD) }\end{array} & \begin{array}{l}\text { heavy drinking occasions, the higher the alco- } \\ \text { hol-related disease burden. }\end{array}\end{array}$

Drinking daily The fewer occasions in which a given amount of alcohol is consumed, the more detrimental the consequences [36]. Thus, given a fixed average volume of consumption, the higher the proportion of daily drinking, the lower the expected burden.

Drinking with Drinking with meals has been shown to be meals less detrimental than drinking at other times. [37]. Thus, ceteris paribus, the higher the proportion of alcohol consumed with meals, the lower the alcohol-related disease burden.

Drinking in public places

Drinking in public often requires transportation, and thus has been linked to traffic accidents and injuries [38]. Also, there may be psychological consequences like risky shift. Thus, the higher the proportion of alcohol consumed in public, the higher the alcohol-related disease burden. Again, this holds only when volume and other influencing factors are held constant.

* This criterion was not used by Gmel et al. [4] since the information was not available.

greater risk of injury than those who did not engage in RSOD. Moreover, those who had overall low consumption, but occasionally engaged in RSOD, were at higher risk of alcohol-related injury compared with chronic heavy drinkers who consumed much more alcohol overall, possibly due to the high tolerance for alcohol among chronic heavy drinkers.

Research has also examined the relationship between the overall drinking pattern of drinkers in a country ('country level drinking pattern') and health risks. Norström [10] reported that the added contribution to total mortality (all causes) from an extra liter of alcohol per capita is much larger in countries where drinking is infrequent but typically takes the form of RSOD, compared to countries where drinking is frequent but typically consumed in moderate amounts. This suggests that, at equal levels of overall volume of consumption, countries with a predominant pattern of infrequent consumption but recurrent RSOD will experience a higher rate of alcoholrelated deaths due to acute outcomes (accident, suicide, homicide) compared to countries with a pattern of frequent consumption but of moderate amounts of alcohol. Similar findings regarding cultural differences were reported by the European Comparative Alcohol Study [11], using aggregated data from 15 European countries. In this study, people in Southern Europe, where the use of alcohol is integrated into everyday life, reported harm only at higher levels of volume consumption, while those in Northern Europe, where alcohol consumption is infrequent but RSOD is common, reported harm even at low levels, with harm increasing more strongly in Northern than in Southern European countries for each additional liter of alcohol.

Although RSOD is the most studied aspect of drinking pattern, there are aspects of drinking pattern other than RSOD that may modify the risks for consequences. These include drinking location (e.g. in public places vs. routinely with meals) and usual frequency and quantity of consumption. A first attempt to construct a composite measure of drinking pattern using several indicators of drinking pattern was made in the comparative risk analysis (CRA) done as part of the 2000 Global Burden of Disease (GBD) study $[1,12]$. In these analyses, a detrimental drinking pattern was defined as more frequently (1) consuming alcohol in higher quantities per occasion, (2) drinking to intoxication, (3) festive drinking and drinking in public places, and (4) less frequent daily drinking and drinking with meals [13] (see table 1 for the rationale of these criteria). The scale was validated by analysis at the aggregate country level, e.g. showing a beneficial effect on coronary heart disease mortality for countries with the most beneficial pattern of drinking and increasingly detrimental effects of drinking for countries with more detrimental drinking patterns [14], as well as a higher rate of alcohol-related injuries presenting to emergency departments in countries with a more detrimental drinking pattern [7]. CRA provides insight into the potential impact of drinking pattern but is limited in that it defines a detrimental drinking pattern as a country-level variable mostly using data from key informants [15] and did not address the relationship between detrimental drinking pattern and negative consequences from drinking at the level of the individual drinker.

Further research on country-level drinking patterns based on the CRA criteria described in table 1 was done by $\mathrm{Gmel}$ et al. [4] using individual survey data (rather 
than key informant reports) to derive country drinking pattern scores. Results showed that countries rank differently on a drinking pattern scale depending on whether drinking pattern is defined by the drinking habits of men or women, and that key informant ratings from previous CRA reflected mainly men's drinking patterns. These analyses were important in identifying the importance of gender differences, but as with previous CRA, this study did not analyze whether drinking pattern was associated with negative consequences at the level of the individual drinker. The present study addresses a number of issues that emerged from previous research on drinking patterns.

First, the study examines the relationship between drinking pattern and negative consequences of drinking both at the level of the individual drinker and at the country level, i.e. the extent that consequences are influenced by the drinking pattern of the individual drinker as well as the sociocultural context for consumption (e.g. a general pattern of drinking to intoxication in the culture) [16].

Second, the study uses several criteria (based on CRA) for defining drinking pattern, including not only RSOD, but also usual frequency and amount consumed, drinking in public places, and drinking with meals.

Third, analyses of the relationship between drinking pattern and negative consequences are made separately for men and women because country differences in the relationship between drinking pattern and negative consequences for drinking may differ for men and women.

Fourth, the importance of drinking pattern is likely dependent on the nature of the consequences. Specifically, two types of alcohol-related consequences have been distinguished: (1) those mainly related to the longterm or chronic effects of alcohol use (e.g. liver cirrhosis, dependence symptoms) which would be expected to be strongly related to volume and less affected by drinking pattern, and (2) those related to short-term or acute effects of alcohol use [17], such as injuries (unintentional and intentional) [18], for which drinking pattern would be expected to play a more important role [15].

The aim of the present research is to determine the extent that drinking pattern is associated with the type of negative consequences experienced from drinking (i.e. fights and injuries vs. symptoms of alcohol use disorder) over and above the total alcohol the person usually consumes, whether this association is similar for men and women across different countries, and the relative influence on this association of individual-level and countrylevel drinking patterns.

Drinking Pattern and Alcohol-Related Negative Consequences

\section{Methods}

\section{Data}

These analyses used data from the Gender, Alcohol and Culture: An International Study (GENACIS) project $[19,20]$. The study included 45 surveys from more than 35 countries (for more details visit www.genacis.org). Analyses for the present study were limited to 18 countries that provided sufficient data on the 5 aspects used by Gmel et al. [4] to determine the drinking pattern scores (table 1) and alcohol-related consequences. The countries considered for this study were Argentina, Australia, Belize, Costa Rica, Czech Republic, Denmark, Finland, Hungary, Iceland, Isle of Man, Japan, Nicaragua, Nigeria, Peru, Sri Lanka, Sweden, Uganda and Uruguay. Eleven countries provided data of nationally representative surveys, whereas data from Argentina, Australia, Sri Lanka, Nicaragua, Nigeria, Peru and Uganda were based on regional surveys. Although the sampling frame affects the generalizability of findings to that country, it does not affect the current research question addressing individual and cultural level drinking patterns. Surveys were conducted between 2000 and 2007, generally in face-to-face interviews, with other fieldwork methods used in Australia, Denmark and Sweden (telephone survey), Japan (postal), and Iceland and Isle of Man (combination of telephone and postal). Table 2 presents an overview of the surveys and their characteristics.

The total sample size of each country and the rate of current drinkers stratified by gender are shown in table 2 . For the present study, the sample was restricted to current drinkers and age range was restricted to 20-65 years of age for greater comparability across surveys.

\section{Independent Variables}

Daily Volume of Drinking. This indicator was based on the annual frequency of drinking occasions multiplied by the usual quantity in grams of pure ethanol divided by 365 days. Volume is reported in the number of drinks per day ( 1 drink $=10 \mathrm{~g}$ of pure ethanol). In 14 countries (Argentina, Belize, Costa Rica, Czech Republic, Denmark, Finland, Hungary, Iceland, Nicaragua, Nigeria, Peru, Sweden, Uganda and Uruguay), this measure was based on beverage-specific questions; however, we used the generic measure to calculate volume in 4 countries either because it was the only measure available (Australia, Japan) or because it resulted in higher volumes than the beverage-specific measure (Isle of Man, Sri Lanka).

Drinking Pattern Score. The drinking pattern score was calculated from frequency of drinking, average quantity per occasion, frequency of RSOD, frequency of drinking with meals and frequency of drinking in public. The score was calculated based on the approach of Gmel et al. [4] in which all indicators were dichotomized between a more detrimental drinking pattern (1) and a less detrimental drinking pattern (0). The indicators used to construct the scale are described below.

Annual Overall Frequency of Drinking (Daily Less Detrimental, Less than Daily Coded as More Detrimental). Respondents were asked to report their usual frequency of drinking based on reported overall frequency or beverage-specific frequency. The maximum frequency reported for a specific beverage or for drinking overall was used. An individual scored 1 on this measure if the drinking frequency was less than 312 drinking days (equivalent to 6 drinking days per week), and 0 otherwise. 
Table 2. Survey information, total sample sizes and proportion of drinkers aged 20-65

\begin{tabular}{|c|c|c|c|c|c|c|c|c|}
\hline & Sampling & Survey mode & $\begin{array}{l}\text { Survey } \\
\text { year }\end{array}$ & $\begin{array}{l}\text { RSOD } \\
\text { cut-off, g }\end{array}$ & $\begin{array}{l}\text { Men } \\
\%\end{array}$ & $\begin{array}{l}\text { Women } \\
\%\end{array}$ & $\begin{array}{l}\text { Men } \\
\mathrm{n}\end{array}$ & $\begin{array}{l}\text { Women } \\
\mathrm{n}\end{array}$ \\
\hline Argentina & regional & face-to-face & 2003 & $60+$ & 92.8 & 77.6 & 372 & 575 \\
\hline Australia & regional & telephone & 2007 & $60+$ & 88.4 & 78.0 & $401^{\mathrm{a}}$ & $596^{\mathrm{a}}$ \\
\hline Belize & national & face-to-face & 2005 & $50+$ & 51.2 & 19.4 & 1,604 & 1,790 \\
\hline Costa Rica & national & face-to-face & 2003 & $60+$ & 67.3 & 42.4 & 357 & 718 \\
\hline Czech Republic & national & face-to-face & 2002 & $90+$ & 90.4 & 79.9 & 1,172 & 1,201 \\
\hline Denmark & national & telephone survey & 2003 & $72+$ & 96.9 & 92.0 & 698 & 878 \\
\hline Finland & national & $\begin{array}{l}\text { face-to-face (AUDIT: } \\
\text { self-administration) }\end{array}$ & 2000 & $60+$ & 92.2 & 90.2 & 839 & 839 \\
\hline Hungary & national & $\begin{array}{l}\text { face-to-face (self- } \\
\text { administered questionnaire } \\
\text { for alcohol questions) }\end{array}$ & 2001 & $60+$ & 90.6 & 74.9 & 1,074 & 1,177 \\
\hline Iceland & national & $\begin{array}{l}\text { half postal and half } \\
\text { telephone survey }\end{array}$ & 2001 & $65+$ & 87.0 & 84.7 & 981 & 1,102 \\
\hline Isle of Man & national & $\begin{array}{l}57.5 \% \text { face-to-face and } \\
42.5 \% \text { telephone) }\end{array}$ & 2005 & $40+$ & 92.9 & 86.3 & 360 & 432 \\
\hline Japan & national & postal & 2001 & $72+$ & 91.1 & 77.0 & 1,031 & 1,048 \\
\hline Nicaragua & regional & face-to-face & 2005 & $60+$ & 43.3 & 10.5 & 546 & 1,291 \\
\hline Nigeria & regional & face-to-face & 2003 & $60+$ & 42.1 & 22.3 & 1,060 & 905 \\
\hline Peru & regional & face-to-face & 2005 & $60+$ & 81.5 & 59.9 & 481 & 945 \\
\hline Sri Lanka & regional & face-to-face & 2002 & $60+$ & 53.6 & 6.4 & 513 & 533 \\
\hline Sweden & national & telephone & 2002 & $72+$ & 88.4 & 79.1 & $711^{\mathrm{a}}$ & $709^{\mathrm{a}}$ \\
\hline Uganda & regional & face-to-face & 2003 & $60+$ & 51.2 & 39.5 & 682 & 706 \\
\hline Uruguay & national & face-to-face & 2004 & $80+$ & 81.1 & 60.3 & 349 & 592 \\
\hline
\end{tabular}

AUDIT $=$ Alcohol Use Disorder Identification Test.

a This number represents only the subsample that answered the questions about drinking indicators and questions regarding alcohol-related consequences.

Average Quantity per Occasion. This indicator was obtained by dividing the annual volume by the annual frequency of drinking occasions. When the quantity exceeded $40 \mathrm{~g}$ of pure ethanol, individuals were scored 1 , and 0 otherwise.

Frequency of RSOD. This indicator was defined by the annual frequency of drinking a particularly high amount of alcohol on a single occasion (e.g. 5 drinks or more). The amount of alcohol consumed on such an occasion was generally $60+\mathrm{g}$ of pure alcohol (table 2). In 7 countries (Belize, Czech Republic, Denmark, Finland, Iceland, Peru, Sweden), the indicator was derived using a single question. For the remaining countries, the measure was derived from the graduated frequency measure [21] which asks the respondent first to identify the highest amount of alcohol consumed in the past 12 months. This maximum quantity serves as a 'starting point' for questions on graduated amounts of alcohol, starting with the frequency of drinking $12+$ amounts, and continuing for each descending quantities (8-11 drinks, 5-7 drinks, 3-4 drinks and 1-2 drinks). The frequency of RSOD used in the present study was constructed by summing the frequency of all drinking quantities beyond 4 glasses. An individual scored 1 on this indicator if the RSOD occurred at least half of the times when drinking alcohol (ratio $>0.5$ ), and 0 otherwise.

Annual Frequency of Drinking with Meals and Drinking in Public Places. These were coded based on questions regarding the frequency of drinking in different contexts, e.g. 'Thinking back over the past 12 months, about how often did you drink in the following circumstances? - at a meal'. An individual received a score of 1 if drinking with meals occurred in less than $50 \%$ of the overall drinking occasions (ratio $<0.5$ ) and 0 otherwise. The measure of drinking in public places was derived from questions on the frequency of drinking in four locations (at home, at a friend's home, in a bar/pub/disco and in a restaurant). The ratio of the frequency of at-home drinking to the sum of all four frequencies was used as indicator for 'at home drinking'. An individual scored 1 if the ratio was smaller than 0.5 (i.e. more than $50 \%$ of all drinking situations took place outside the home), and 0 otherwise.

\section{Dependent Variables}

The present study included two groups of alcohol-related consequences: (1) alcohol use disorder symptoms (AUDS) and (2) alcohol-related injuries and fights. AUDS were measured using four items commonly used in screening instruments for alcohol dependence and harmful use, such as the Alcohol Use Disorder Identification Test [22] or CAGE [23], with a reference period of the last 12 months. These included: (1) 'Have you been unable to remember what happened the night before because you had been drinking?', (2) 'Have you found you were not able to stop drinking once you started?', (3) 'Have you needed a first drink in the morn- 
ing to get yourself going after a heavy drinking session', and (4) 'Have you had any feelings of guilt or remorse after drinking?'. An individual scored 1 if at least one of these occurred in the last 12 months, and 0 otherwise.

For alcohol-related injuries and fights, the indicator was based on two questions: (1) 'Have you or someone else been injured as a result of your drinking?', and (2) 'Have you gotten into a fight while drinking?'. An individual scored 1 if one or both of these occurred in the last 12 months, and 0 if neither occurred.

\section{Statistical Analysis}

To construct the drinking pattern score, a categorical principal component analysis [24], also called optimal scaling, was used to scale countries according to their drinking patterns using individual survey data separately for each sex. To adjust for the fact that countries with a larger sample size would have a greater effect on the overall drinking pattern score, samples were weighted so that each country contributed to the scale in the same way. The weighted sample size was arbitrarily set to 500 for each gender in each country. To obtain a single aggregated country drinking pattern score by sex (table 3), the individual scale values were averaged, stratified by country and sex.

To estimate the impact of drinking pattern on alcohol-related consequences, regression models using the multilevel software package HLM 6.06 [25] were analyzed. AUDS and alcohol-related injuries and fights served as binary outcomes, with individual drinking pattern scores and the daily volume of alcohol intake (given in number of drinks per day) used as independent variables on the individual level. All regression models were done separately for men and women, and the age of respondents was included as a covariate.

Three multi-level regression models with increasing complexity [26] were estimated. In the first model, the impact of individual volume of alcohol and the individual drinking pattern score on alcohol-related consequences were estimated in a random slope intercept model across the 18 countries (without any country level variables). For the second model, the mean of the drinking pattern scores for the country was included as a second level variable. A third model evaluated the cross-level interaction between the individual volume of alcohol consumption and the country-level drinking pattern score (i.e. the extent that the variation of slopes across countries was explained by the gender-specific country-level drinking pattern score).

\section{Results}

The aggregated drinking pattern scores are shown in table 3. As shown by the final column in the table, rank order from least to most detrimental drinking across countries corresponds quite well with the CRA pattern scores originally calculated as part of the GBD study [1]. For example, Belize and Nicaragua showed the most detrimental drinking patterns for men and women based on both the present analyses and CRA scores, while at the other extreme, Japan, followed by Argentina, Denmark and Uruguay, had the least detrimental pattern scores.
Table 3. Drinking pattern scores and ranking of countries based on individual level analysis of drinkers only and country CRA scores from Rehm et al. [1]

\begin{tabular}{|c|c|c|c|c|c|}
\hline & \multicolumn{2}{|l|}{ Men } & \multicolumn{2}{|l|}{ Women } & \multirow{2}{*}{$\begin{array}{l}\text { Country } \\
\text { CRA } \\
\text { scores }\end{array}$} \\
\hline & $\begin{array}{l}\text { drinking } \\
\text { pattern } \\
\text { score }\end{array}$ & rank & $\begin{array}{l}\text { drinking } \\
\text { pattern } \\
\text { score }\end{array}$ & rank & \\
\hline Japan & -0.93 & 1 & -0.56 & 2 & 1 \\
\hline Argentina & -0.62 & 2 & -0.57 & 1 & 2 \\
\hline Denmark & -0.49 & 3 & -0.17 & 8 & 2 \\
\hline Uruguay & -0.43 & 4 & -0.45 & 3 & 3 \\
\hline Hungary & -0.28 & 5 & -0.29 & 5 & 3 \\
\hline Czech Republic & -0.28 & 6 & 0.05 & 12 & 2 \\
\hline Finland & -0.18 & 7 & -0.22 & 6 & 3 \\
\hline Isle of Man & -0.13 & 8 & -0.20 & 7 & 2 \\
\hline Sweden & -0.12 & 9 & -0.07 & 10 & 3 \\
\hline Australia & 0.04 & 10 & -0.12 & 9 & 2 \\
\hline Iceland & 0.12 & 11 & 0.33 & 15 & 3 \\
\hline Nigeria & 0.16 & 12 & 0.45 & 16 & 2 \\
\hline Costa Rica & 0.27 & 13 & -0.01 & 11 & 4 \\
\hline Sri Lanka & 0.29 & 14 & -0.32 & 4 & 3 \\
\hline Uganda & 0.30 & 15 & 0.29 & 14 & 3 \\
\hline Peru & 0.39 & 16 & 0.17 & 13 & 3 \\
\hline Belize & 0.88 & 17 & 0.63 & 17 & 4 \\
\hline Nicaragua & 0.96 & 18 & 0.97 & 18 & 4 \\
\hline
\end{tabular}

Negative pattern scores and lower CRA scores show a less detrimental drinking pattern; positive pattern scores and higher CRA scores show a more detrimental pattern. Rankings are from least to most detrimental drinking pattern.

The correlation between the CRA detrimental pattern score and the estimated aggregated scale was $r=0.78$ for men and $\mathrm{r}=0.57$ for women. As shown in the table, although the correlation between rankings for male and female drinking was high $(\mathrm{r}=0.86)$, the pattern of rankings differs to some extent for men and women. For example, male drinkers from Sri Lanka had one of the most detrimental drinking pattern scores (rank 14), while the scores for female drinkers from Sri Lanka ranked among the least detrimental (rank 4).

The results of regression models calculated separately by gender and type of consequence are shown in table 4 . The first set of models (model 1) of the two indicators of alcohol-related consequences revealed that for both genders, the risk for AUDS and alcohol-related injuries/fights increased with increasing individual volume and with higher drinking pattern scores. When the country drinking pattern score was included in the model as an effect on the intercept (model 2), the effect of the individual's 
Table 4. Hierarchical linear models (1-3) of the regression of AUDS and injuries/fights on individual-level drinking pattern score (DPS), usual volume measured in drinks per day (volume $\mathrm{d} / \mathrm{d}$ ), gender-specific country drinking pattern score effect on intercept (DPS mean 1), and the country drinking pattern score effect on the slope of individual volume (DPS mean 2)

\begin{tabular}{|c|c|c|c|c|c|c|c|c|c|c|c|c|}
\hline \multirow{3}{*}{$\begin{array}{l}\text { Individual/ } \\
\text { country }\end{array}$} & \multicolumn{6}{|l|}{ AUDS } & \multicolumn{6}{|c|}{ Injuries and fights } \\
\hline & \multicolumn{2}{|l|}{ model 1} & \multicolumn{2}{|l|}{ model 2} & \multicolumn{2}{|l|}{ model 3} & \multicolumn{2}{|l|}{ model 1} & \multicolumn{2}{|l|}{ model 2} & \multicolumn{2}{|l|}{ model 3} \\
\hline & odds & $\mathrm{CI}$ & odds & $\mathrm{CI}$ & odds & $\mathrm{CI}$ & odds & CI & odds & $\mathrm{CI}$ & odds & CI \\
\hline \multicolumn{13}{|l|}{ Men } \\
\hline Intercept & $0.47^{* * *}$ & $0.32,0.71$ & $0.47^{* * *}$ & $0.32,0.69$ & $0.47^{* * *}$ & $0.33,0.68$ & $0.14^{* * *}$ & $0.09,0.22$ & $0.14^{* * *}$ & $0.09,0.21$ & $0.14^{* * *}$ & $0.09,0.21$ \\
\hline DPS mean 1 & & & $2.90^{* * *}$ & $1.72,4.91$ & $2.17^{*}$ & $1.11,4.24$ & & & $1.55^{* *}$ & $1.14,2.11$ & 1.35 & $0.88,2.08$ \\
\hline DPS & $1.38^{* * *}$ & $1.24,1.54$ & $1.39^{* * *}$ & $1.25,1.55$ & $1.39^{* * *}$ & $1.25,1.54$ & $1.23^{* * *}$ & $1.11,1.37$ & $1.24^{* * *}$ & $1.12,1.36$ & $1.24^{* * *}$ & $1.12,1.37$ \\
\hline Volume $\mathrm{d} / \mathrm{d}$ & $1.35^{* * *}$ & $1.20,1.51$ & $1.34^{* * *}$ & $1.20,1.50$ & $1.34^{* * *}$ & $1.21,1.49$ & $1.17^{* * *}$ & $1.11,1.24$ & $1.17^{* * *}$ & $1.12,1.23$ & $1.17^{* * *}$ & $1.12,1.23$ \\
\hline DPS mean 2 & & & & & 0.88 & $0.77,1.00$ & & & & & 0.97 & $0.92,1.03$ \\
\hline \multicolumn{13}{|l|}{ Women } \\
\hline Intercept & $0.13^{* * *}$ & $0.08,0.21$ & $0.13^{* * *}$ & $0.08,0.20$ & $0.13^{* * *}$ & $0.08,0.20$ & $0.04^{* * *}$ & $0.02,0.06$ & $0.04^{* * *}$ & $0.02,0.06$ & $0.04^{* * *}$ & $0.02,0.06$ \\
\hline DPS mean 1 & & & $2.77^{*}$ & $1.05,7.34$ & 2.26 & $0.87,5.90$ & & & $2.59^{*}$ & $1.16,5.80$ & $2.56^{*}$ & $1.13,5.78$ \\
\hline DPS & $1.56^{* * *}$ & $1.31,1.86$ & $1.55^{* * *}$ & $1.31,1.85$ & $1.56^{* * *}$ & $1.31,1.84$ & $1.42^{* * *}$ & $1.26,1.59$ & $1.42^{* * *}$ & $1.30,1.56$ & $1.42^{* * *}$ & $1.29,1.55$ \\
\hline Volume d/d & $1.78^{* * *}$ & $1.45,2.18$ & $1.77^{* * *}$ & $1.44,2.17$ & $1.78^{* * *}$ & $1.49,2.12$ & $1.39^{* * *}$ & $1.24,1.58$ & $1.38^{* * *}$ & $1.23,1.55$ & $1.40^{* * *}$ & $1.26,1.56$ \\
\hline DPS mean 2 & & & & & $0.75^{*}$ & $0.57,0.97$ & & & & & $0.79^{* * *}$ & $0.69,0.91$ \\
\hline
\end{tabular}

Model 1 includes only the individual drinking pattern and volume scores; model 2 includes the individual drinking pattern and volume scores and the country drinking pattern score as an effect on the intercept; model 3 includes the individual drinking pattern and volume scores and the country drinking pattern score as an effect on the intercept and the slope. All models were adjusted for age. ${ }^{*} \mathrm{p}<0.05 ;{ }^{* *} \mathrm{p}<0.01 ;{ }^{* * *} \mathrm{p}<0.001$.

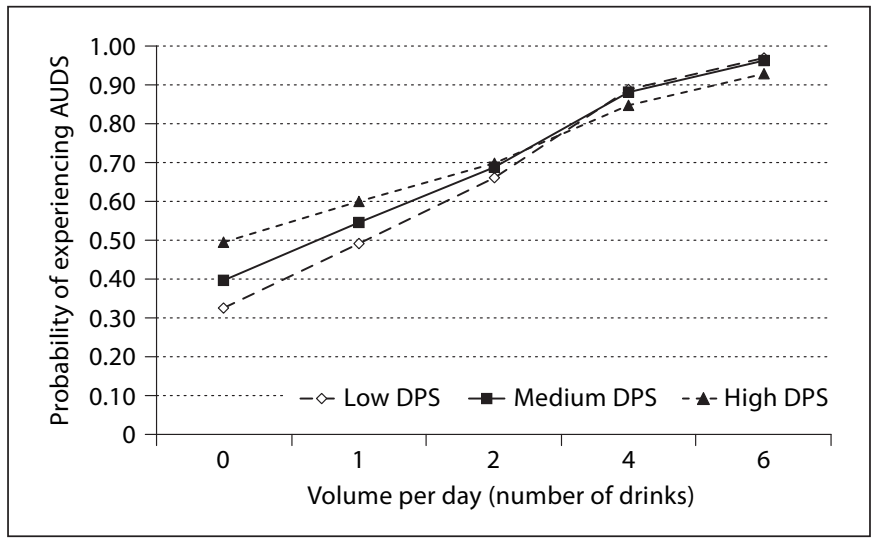

Fig. 1. Relationship of probability of experiencing AUDS and volume of consumption by high, medium and low detrimental drinking pattern score (DPS) countries for women. Models were fitted at the mean age of 42 years and the mean individual DPS. Low, medium and high DPS refer to the 6 countries with the lowest DPS, 6 countries with medium and 6 countries with the highest DPS, based on their DPS country mean.

volume and drinking pattern score remained unchanged. Nevertheless, the country-level mean drinking pattern score significantly increased risk of both AUDS and injuries/fights over and above the effect found for individual level drinking pattern and volume.
A third set of regression models (model 3 in table 4) evaluated the impact of the gender-specific country-level mean drinking pattern score on the link between the individual measure of volume per day and alcohol-related consequences. For both types of consequences, an odds ratio under 1 was found (significant only for women); that is, the higher the country-level drinking pattern score, the weaker the association between volume of drinking by the individual and negative consequences from drinking. To better understand the meaning of this effect, a graph of the predicted probability of experiencing AUDS among women by countries with different levels of detrimental drinking pattern can be seen in figure 1. To calculate this graph, countries were categorized as being low drinking pattern score countries (6 lowest scoring countries), medium drinking pattern score countries (middle 6) and high drinking pattern score countries (highest 6). As shown in figure 1, the intercept of the high drinking pattern score countries was larger than for the medium and low drinking pattern score countries at low volumes of consumption, while the scores were relatively similar at high volumes of consumption. That is, the slope of the relationship between volume and probability of experiencing AUDS was flatter for high drinking pattern score countries and steeper for countries with lower drinking pattern scores, indicating that low drinking pattern score countries were less likely to report AUDS at low con- 
sumption levels than high drinking pattern score countries; however, at higher volumes, rates of AUDS were similar regardless of country-level drinking pattern score.

\section{Discussion}

These results confirm and extend previous research on drinking pattern. First, results based on survey data corresponded quite well with the CRA drinking pattern scores which were used in the GBD study [1], which was based mostly on reports of key informants. However, as found in previous analyses comparing survey data to original CRA drinking pattern scores [4], the CRA drinking pattern scores were more strongly related to drinking by men than by women in the country. Correspondingly, rankings varied somewhat by gender, suggesting that men and women do not necessarily have the same predominant drinking patterns within the same country. It is thus important for cross-cultural research to estimate a country's drinking pattern separately for men and women.

The hierarchical linear model results were consistent with the expectation that the higher the amount of alcohol consumed, the higher the risk for negative consequences from drinking, including both injuries/fights and AUDS. The findings also confirm previous research on the impact of RSOD [9], which found that a detrimental drinking pattern contributes to negative consequences from drinking over and above the effect of volume. Although it might be expected that drinking pattern would be a more important predictor of acute consequences, such as injury/fight rather than AUDS, our analyses suggest that drinking pattern is associated with both types of consequences. One reason for this finding may be that AUDS available for analyses did not necessarily reflect effects of chronic drinking only, but also acute consequences when asked in general population surveys, especially the items of 'drinking to get over the effects of a RSOD session' and experiencing 'guilt and remorse from drinking'.

The results pertaining to country-level drinking patterns are especially important because this is the first study which has simultaneously examined the relationship between drinking pattern and negative consequences from drinking at both the individual and the country level. The analyses indicated that the country's drinking pattern had an additional impact on the general level (represented by the intercepts) of risks for alcohol-related consequences, independent of the individual's alcohol consumption (including both volume and patterns). In other words, respondents reported more alcohol-related consequences from their drinking in countries with a generally more detrimental pattern of drinking. This effect is over and above the relationship between individual drinking pattern and alcohol-related consequences. One interpretation of this finding is that experiencing negative alcohol-related consequences does not only depend on the individual's drinking behavior (volume/pattern), but also on environmental and societal factors, such as how people in the country generally drink, drinking culture and drinking habits which may influence the likelihood of consequences, such as being involved in a fight while drinking [7].

It should be noted that country differences in reporting drinking consequences may also be influenced by the position of drinking in the culture and the expectations about drunken behaviors $[27,28]$. Thus, the higher rate of alcohol-related consequences in countries with a more detrimental drinking pattern may be partly attributable to a greater willingness in these cultures to admit alcohol-related consequences, perhaps because infrequent RSOD is seen as an acceptable time-out behavior leading to excusable consequences [28] compared with self-reports in countries where drinking is frequent and integrated in the culture (e.g. drinking with meals), but where RSOD is often less tolerated.

In addition, some studies have shown that the attribution of alcohol as a cause for consequences varies across countries $[29,30]$. Similarly, studies have shown that reporting of AUDS varies independently of volume of drinking [31]. The present study would then suggest that a more detrimental drinking pattern on country level may partly be associated with a higher willingness to report alcohol-related consequences as well as a greater likelihood of linking problems to drinking, independent of whether these consequences are short-term or longterm.

An important finding of the present research was the moderating effect of the country's drinking pattern on the individual's volume of alcohol consumption, especially for women. Specifically, in countries with a more detrimental pattern of drinking, women reported more alcohol-related consequences at a low volume compared to those in countries with a less detrimental pattern. However, for individuals who reported a high volume of drinking, there was little or no effect from the countrylevel drinking pattern. Research has shown that attitudes toward alcohol consumption can have a strong influence 
on perceived alcohol-related consequences, such as feelings of guilt, especially for women [31]. Therefore, one possible interpretation of this interaction is that even light drinking among women can be perceived as problematic in cultures where drinking is relatively rare and typically consumed in large amounts. This is consistent with previous findings [11] using aggregated data from 15 European countries that found that harm was experienced even at low levels of alcohol consumption in countries with a pattern of RSOD, but only at high levels in countries where alcohol consumption was more integrated into everyday life.

Although this research suggests important impacts of drinking pattern at both the individual and country level, it also has some limitations. When interpreting the findings of cross-country survey research on the alcoholconsequence link using self-reported data, possible confounding effects from different sources should be considered. These include: (1) effects that are due to different norms and interpretations across countries of whether a consequence is alcohol-related or not (e.g. subjective attribution at the individual and societal level), (2) effects due to real differences in alcohol use (e.g. more heavy drinkers across countries resulting in higher prevalence rates of AUDS), and (3) real effects at the societal level (e.g. increased risk of certain alcohol-related consequences such as injuries that are not only due to individual drinking but also related to the drinking of others). Future research is needed that incorporates other measures of alcohol-related consequences such as hospitalization or treatment data to better disentangle these different effects.

Other limitations of the study include differences in the method of data collection among countries, different measures used (e.g. for assessing alcohol consumption and RSOD) and the small sample sizes for some countries. Still, it has been argued that while prevalence estimates might be strongly affected by methodological differences, this is less likely when comparing relationships between drinking and other variables across countries [32]. The present study, despite some methodological shortcomings, showed strong agreement with other research using different methodologies (such as in the CRA exercise [Eur Addict Res Vol. 7, No. 3, 2001] or other data sources such as data from emergency departments [30, 33]), consistent with the interpretation that the present findings reflect valid relationships between individual and country-level drinking patterns and negative consequences from drinking.

\section{Acknowledgements}

This paper is part of the GENACIS project. GENACIS is a collaborative international project affiliated with the Kettil Bruun Society for Social and Epidemiological Research on Alcohol, coordinated by GENACIS partners from the University of North Dakota, the University of Southern Denmark, the Charite University Medicine Berlin, the Pan American Health Organization and the Swiss Institute for the Prevention of Alcohol and Drug Problems. Support for aspects of the project comes from the World Health Organization, the Quality of Life and Management of Living Resources Programme of the European Commission (Concerted Action QLG4-CT-2001-0196), the US National Institute on Alcohol Abuse and Alcoholism/National Institutes of Health (Grant No. R21 AA012941 and R01 AA015775), the German Federal Ministry of Health, the Pan American Health Organization and Swiss national funds. Support for individual country surveys was provided by government agencies and other national sources. The study leaders and funding sources for data sets used in this report are: Myriam Munné, Argentina, WHO; Paul Dietze, Australia, grant from National Health and Medical Research Council (398500); Claudina Cayetano, Belize, PAHO; Julio Bejarano, Costa Rica, WHO; Ladislav Csémy, Czech Republic, Ministry of Health (grant No. MZ 23752); Kim Bloomfield, Denmark, Sygekassernes Helsefond, Danish Medical Research Council; Pia Mäkelä, Finland, National Research and Development Centre for Welfare and Health (STAKES); Zsuzsanna Elekes, Hungary, Ministry of Youth and Sport; Hildigunnur Ólafsdóttir, Iceland, Alcohol and Drug Abuse Prevention Council, Public Health Institute of Iceland; Moira and Martin Plant, Isle of Man, Isle of Man Medical Research, University of the West of England, Bristol; Shinji Shimizu, Japan, Japan Society for the Promotion of Science (grant No. 13410072); Jose Trinidad Caldera, Nicaragua, PAHO; Akanidomo Ibanga, Nigeria, WHO; Marina Piazza, Peru, PAHO; Siri Hettige, Sri Lanka, WHO; Karin Bergmark, Sweden, Ministry for Social Affairs and Health; Nazarius Mbona Tumwesigye, Uganda, WHO; Raquel Magri, Uruguay, WHO.

\section{References}

1 Rehm J, Room R, Monteiro MG, Gmel G, Graham K, Rehn N, Sempos CT, Frick U, Jernigan D: Alcohol use; in Ezzati M, Lopez AD, Rodgers A, Murray CJL (eds): Comparative Quantification of Health Risks Global and Regional Burden of Disease Attributable to Selected Major Risk Factors, vol 1. Geneva, WHO, 2004, pp 959-1108.

2 Rehm J, Mathers C, Popova S, Thavorncharoensap M, Teerawattananon Y, Patra J: Global burden of disease and injury and economic cost attributable to alcohol use and alcohol use disorders. Lancet 2009;373:22232233.

3 Rehm J, Ashley MJ, Room R, Single E, Bondy SJ, Ferrence RG, Giesbrecht N: On the emerging paradigm of drinking patterns and their social and health consequences. Addiction 1996;91:1615-1621. 
-4 Gmel G, Room R, Kuendig H, Kuntsche S: Detrimental drinking patterns: empirical validation of the pattern values score of the Global Burden of Disease 2000 study in 13 countries. J Subst Use 2007;12:337-358.

5 Wechsler H, Austin SB: Binge drinking: the five/four measure. J Stud Alcohol 1998;59: 122-124.

6 Wright NR, Cameron D: A pilot study of prospectively recorded drinking patterns among British men who habitually drink 1-4 units of alcohol per day. Alcohol Alcohol 1997;32: 777-778.

7 Anderson P: Binge Drinking and Europe. London, Institute of Alcohol Studies, 2007.

$\checkmark 8$ Dawson DA, Li TK, Grant BF: A prospective study of risk drinking: at risk for what? Drug Alcohol Depend 2008;95:62-72.

-9 Gmel G, Bissery A, Gammeter R, Givel JC, Calmes JM, Yersin B, Daeppen JB: Alcoholattributable injuries in admissions to a Swiss emergency room - an analysis of the link between volume of drinking, drinking patterns and preattendance drinking. Alcohol Clin Exp Res 2006;30:501-509.

10 Norström T: Per capita alcohol consumption and all-cause mortality in 14 European countries. Addiction 2001;96:S113-S128.

11 Ramstedt M: Alcohol-related mortality in 15 European countries in the postwar period; in Norström $\mathrm{T}$ (ed): Alcohol in Postwar Europe: Consumption, Drinking Patterns, Consequences and Policy Responses in 15 European Countries. Stockholm, Almquist and Wiksell Int., 2002, pp 137-156.

12 Ezzati M, Lopez AD, Rodgers A, Murray CJL: Comparative Quantification of Health Risks. Global and Regional Burden of Disease Attributable to Selected Major Risk Factors. Geneva, WHO, 2004.

-13 Rehm J, Room R, Monteiro MG, Gmel G, Graham K, Rehn N, Sempos CT, Jernigan D: Alcohol as risk factor for global burden of disease. Eur Addict Res 2003;9:157-164.

14 Gmel G, Rehm J, Frick U: Trinkmuster, ProKopf-Konsum von Alkohol und koronare Mortalität. Sucht 2003;49:95-104.

- 15 Rehm J, Monteiro MG, Room R, Gmel G, Jernigan D, Frick U, Graham K: Steps towards constructing a global comparative risk analysis for alcohol consumption: determining indicators and empirical weights for patterns of drinking, deciding about theoretical minimum, and dealing with different consequences. Eur Addict Res 2001;7:138147.
16 Graham K, Leonard KE, Room R, Wild TC, Phil RO, Bois C, Single E: Current directions in research on understanding and preventing intoxicated aggression. Addiction 1998; 39:659-676.

17 World Health Organization (WHO): International Guide for Monitoring Alcohol Consumption and Related Harm. Geneva, WHO, Department of Mental Health and Substance Dependence, Noncommunicable Diseases and Mental Health Cluster, 2000.

18 Gmel G, Rehm J: Harmful alcohol use. Alcohol Res Health 2003;27:52-62.

19 Bloomfield K, Gmel G, Wilsnack SC: Introduction to special issue 'Gender, Culture and Alcohol Problems: A Multi-National Study’. Alcohol Alcohol 2006;41:i3-i7.

-20 Wilsnack RW, Wilsnack SC, Kristjanson AF, Vogeltanz-Holm ND, Gmel G: Gender and alcohol consumption: patterns from the multinational GENACIS project. Addiction 2009;104:1487-1500.

-21 Greenfield TK: Ways of measuring drinking patterns and the difference they make: experience with graduated frequencies. J Subst Abuse 2000;12:33-49.

$\checkmark 22$ Babor TF: Linking science to policy. the role of international collaborative research. Alcohol Res Health 2002;26:66-74.

$\checkmark 23$ Mayfield D, McLeod G, Hall P: The CAGE questionnaire: validation of a new alcoholism instrument. Am J Psychiatry 1974;131: 1121-1123.

24 Bijleveld CJH, van der Kamp LJT, Mooijaart A, van der Kloot WA, van der Leeden R, van der Burg E: Longitudinal Data Analysis: Designs, Models and Methods. London, Sage Publications Ltd., 1998.

25 Raudenbush SW, Bryk AS, Cheong YF, Congdon R, du Toit M: HLM 6: Hierarchical Linear and Nonlinear Modeling. Lincolnwood, SSI Scientific Software International, 2004.

26 Hox JJ (ed): Multilevel Analysis: Techniques and Applications. Mahwah, Lawrence Erlbaum Associates, 2002, p 304.

$\checkmark 27$ Room R, Mäkelä K: Typologies of the cultural position of drinking. J Stud Alcohol 2000;61:475-483.

28 Room R: Intoxication and bad behaviour: understanding cultural differences in the link. Soc Sci Med 2001;53:189-198.

29 Kuendig H, Plant MA, Plant ML, Miller P, Kuntsche S, Gmel G: Alcohol-related adverse consequences: cross-cultural variations in attribution process among young adults. Eur J Public Health 2008;18:386-391.
30 Cherpitel CJ, Bond J, Ye Y, Borges GLG, Room R, Poznyak V, Hao W: Multi-level analysis of causal attribution of injury to alcohol and modifying effects: Data from two international emergency room projects. Drug Alcohol Depend 2006;82:258-268.

-31 Messiah A, Encrenaz G, Sapinho D, Gilbert F, Carmona E, Kovess-Masféty V: Paradoxical increase of positive answers to the Cutdown, Annoyed, Guilt, Eye-opener (CAGE) questionnaire during a period of decreasing alcohol consumption: results from two population-based surveys in Ile-de-France, 1991 and 2005. Addiction 2008;103:598-603.

>32 Bloomfield K, Stockwell T, Gmel G, Rehn N: International comparison of alcohol consumption. Alcohol Res Health 2003;27:95109.

33 Cherpitel CJ, Ye Y, Bond J, Rehm J, Poznyak V, Macdonald S, Stafstrom M, Hao W: Multilevel analysis of alcohol-related injury among emergency department patients: a crossnational study. Addiction 2005;100:18401850.

34 Rossow I, Pernanen K, Rehm J: Accidents, suicide and violence; in Klingemann $\mathrm{H}$, Gmel G (eds): Mapping the Social Consequences of Alcohol Consumption. Dordrecht, Kluwer Academic Publishers, 2001, pp 93-112.

>35 Rehm J, Sempos CT, Trevisan M: Alcohol and cardiovascular disease - more than one paradox to consider. Average volume of alcohol consumption, patterns of drinking and risk of coronary heart disease: a review. J Cardiovasc Risk 2003;10:15-20.

36 Room R, Jernigan D, Carlini-Marlatt B, Gureje O, Mäkelä K, Marshall M, MedinaMora ME, Monteiro MG, Parry CDH, Partanen J, Riley L, Saxena S: Alcohol in Developing Societies: A Public Health Approach. Helsinki, Finnish Foundation for Alcohol Studies in collaboration with World Health Organization, 2002.

37 Ramchandani VA, Kwo PY, Li TK: Effect of food and food composition on alcohol elimination rates in healthy men and women. J Clin Pharmacol 2001;41:1345-1350.

38 Fahrenkrug H, Rehm J: Trinkkontexte und Freizeitaktivitäten in der Vorphase alkoholbezogener Strassenverkehrsunfälle junger Fahrerinen und Fahrer in der Schweiz. Sucht 1995;41:169-180. 